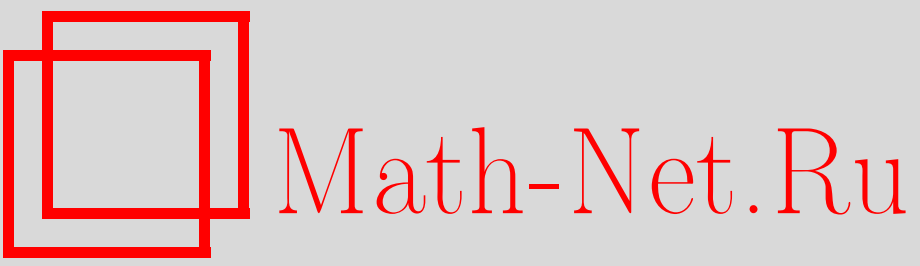

Ю. А. Гладышев, В. В. Калманович, Об использовании матричного метода решения задач теплопроводности в многослойной среде при наличии фазовых переходов, Итоги науки и техн. Сер. Соврем. мат. и ее прил. Темат. обз., 2019, том $172,30-37$

DOI: https://doi.org/10.36535/0233-6723-2019-172-30-37

Использование Общероссийского математического портала Math-Net.Ru подразумевает, что вы прочитали и согласны с пользовательским соглашением

http: //www. mathnet.ru/rus/agreement

Параметры загрузки:

IP : 34.229 .45 .116

26 апреля 2023 г., $17: 10: 12$ 


\title{
ОБ ИСПОЛЬЗОВАНИИ МАТРИЧНОГО МЕТОДА РЕШЕНИЯ ЗАДАЧ ТЕПЛОПРОВОДНОСТИ В МНОГОСЛОЙНОЙ СРЕДЕ ПРИ НАЛИЧИИ ФАЗОВЫХ ПЕРЕХОДОВ
}

\author{
(c) 2019 г. \\ Ю. А. ГЛАДЫШЕВ, В. В. КАЛМАНОВИЧ
}

\begin{abstract}
АннотАция. Работа посвящена возможности применения матричного метода решения уравнения теплопроводности для многослойных сред в случае, когда в каком-либо слое возможен фазовый переход. Рассматриваются только стационарные процессы при отсутствии в среде внутренних источников тепла. Предложен общий метод решения для систем слоев, обладающих сдвиговой, осевой или центральной симметрией, основанный на аппарате обобщенных степеней Берса. По указанному методу проведены вычисления для одного вещества, когда в результате фазового перехода система становится двухслойной. Рассмотрена зависимость координаты точки фазового перехода от внешней температуры, проведено сравнение для сред с вышеперечисленными видами симметрии. Построено поле температур для многослойных сред с различными видами симметрии, когда в каком-либо слое произошел фазовый переход.
\end{abstract}

Ключевые слова: математическая модель, матричный метод, уравнение теплопроводности, многослойная среда, фазовый переход.

\section{ON THE MATRIX METHOD \\ FOR SOLVING HEAT CONDUCTION PROBLEMS \\ IN A MULTILAYER MEDIUM \\ IN THE PRESENCE OF PHASE TRANSITIONS}

\author{
(c) 2019 YU. A. GLADYSHEV, V. V. KALMANOVICH
}

\begin{abstract}
The work is devoted to the applicability of the matrix method for solving the heat equation for multilayer media in the case where a phase transition is possible in some layer. We consider only stationary processes in the absence of internal heat sources. We propose a general method for layer systems with translation, axial, or central symmetry based on the technique of generalized Bers degrees. By the method indicated above, we perform calculations for one substance, when after a phase transition, the system becomes a two-layer system. We consider the dependence of the coordinate of the phase-transition point on the external temperature and compare results obtained for media with types of symmetry indicated above. A temperature field is constructed for multilayer media with various types of symmetry when a phase transition has occurred in a certain layer.
\end{abstract}

Keywords and phrases: mathematical model, matrix method, heat equation, multilayer medium, phase transition.

AMS Subject Classification: 34B05, 34B60, 80A20

Работа выполнена при поддержке Российского фонда фундаментальных исследований (проект № 19-03-00271), а также совместного проекта Российского фонда фундаментальных исследований и правительства Калужской области № 18-41-400001. 
1. Введение. Нелинейные задачи теплопроводности в многослойной среде представляют большой интерес, поскольку в современной технике часто используют материалы, полученные путем соединения различных сред или покрытий, причем при широком диапазоне температур. В многослойной среде возможно наличие слоев, в которых при повышении температуры выше некоторого значения $T_{\phi}$ происходит фазовый переход в состояние с другими параметрами. В слое этот процесс может развиваться постепенно в зависимости от повышения или понижения температуры, пока не установится некоторое конечное стационарное состояние. В данной работе будут рассмотрены только стационарные задачи. Постановка и решение краевых задач в многослойной среде, когда основные параметры не зависят от температуры, изучены в ряде работ (см., например, [5-8]). Авторами был предложен матричный метод построения решения задач тепломассопереноса (см. [2-4]). В настоящей работе показано, что этот метод может быть использован для построения решения, когда в одном или нескольких слоях происходит фазовый переход.

2. Постановка задачи. Рассмотрим процесс теплопроводности в многослойной неоднородной (возможно, искривленной) оболочке, когда в одном из слоев возможен фазовый переход. Предполагая процесс одномерным и направленным по нормали к оболочке, направим ось $x$ по этой нормали. Пусть $x_{1}, \ldots, x_{n+1}$ - координаты слоев, причем $x_{1}$ и $x_{n+1}$ - координаты внешних поверхностей.

Основная система уравнений, определяющая температуру $T^{(i)}(x)$ в $i$-м слое (номер слоя указан в верхнем индексе в скобках и совпадает с номером его меньшей координаты), имеет вид

$$
D_{2}^{(i)} D_{1}^{(i)} T^{(i)}(x)-m_{i}^{2} T^{(i)}(x)=\mu^{(i)}(x), \quad i=\overline{1, n},
$$

где дифференциальные операторы $D_{1}^{(i)}$ и $D_{2}^{(i)}$ определяются выражениями

$$
D_{1}^{(i)}=a_{1}^{(i)}(x) \frac{d}{d x}, \quad D_{2}^{(i)}=a_{2}^{(i)}(x) \frac{d}{d x} .
$$

Для процесса теплопроводности, заданного уравнением $(1)$, функции $a_{1}^{(i)}(x)$ и $a_{2}^{(i)}(x)$ положительны и определяются соотношениями

$$
a_{1}^{(i)}=\lambda^{(i)} x^{s}, \quad a_{2}^{(i)}=x^{-s},
$$

где $\lambda^{(i)}$ - коэффициент теплопроводности $i$-го слоя. Значение показателя $s$ определяется геометрией среды: значение $s=0$ соответствует процессу теплопроводности в среде со сдвиговой симметрией (плоские слои) по оси $x, s=1$-с осевой симметрией (цилиндрические слои) и $s=2-$ с центральной симметрией (сферические слои).

Плотность теплового потока определяется выражением

$$
J^{(i)}(x)=-D_{1}^{(i)} T^{(i)}(x) .
$$

Коэффициенты $m^{(i)}$ позволяют учитывать общий характер процессов (например, наличие внешнего теплообмена и др.). Функция $\mu^{(i)}(x)$ определяет объемную плотность мощности распределения источников.

Далее рассматривается простейший случай процесса теплопроводности, когда $m^{(i)}=0$ и нет внутренних источников, т.е. уравнение (1) принимает вид

$$
D_{2}^{(i)} D_{1}^{(i)} T^{(i)}(x)=0, \quad i=\overline{1, n} .
$$

На внутренних границах слоев приняты условия согласования типа идеального контакта, состоящие в непрерывности температуры и теплового потока:

$$
T^{(i)}\left(x_{i+1}\right)=T^{(i+1)}\left(x_{i+1}\right), \quad J^{(i)}\left(x_{i+1}\right)=J^{(i+1)}\left(x_{i+1}\right), \quad i=\overline{1, n-1} .
$$

Далее предполагается, что для всех слоев коэффициенты теплопроводности $\lambda^{(i)}$ не зависят от температуры, за исключением некоторого $k$-го слоя, на котором $\lambda^{(k)}$ зависит от температуры и определяется следующим образом:

$$
\lambda^{(k)}(T)= \begin{cases}\lambda_{1}^{(k)}, & T<T_{\phi}, \\ \lambda_{2}^{(k)}, & T>T_{\phi} ;\end{cases}
$$


здесь $T_{\phi}$ - температура фазового перехода в $k$-м слое, $\lambda_{1}^{(k)}$ и $\lambda_{2}^{(k)}$ - теплопроводности фаз. В этом случае уравнение (3) нелинейное.

На границах $x_{1}, x_{2}$ системы слоев заданы температуры, т.е. поставлена первая краевая задача

$$
\left.T^{(1)}\right|_{x_{1}}=T_{1},\left.\quad T^{(n)}\right|_{x_{n+1}}=T_{2}
$$

3. Матричный метод построения решения. Решения системы (2)-(3) с краевыми условиями (4) в данной работе строится матричным методом, который был описан нами ранее в ряде работ (см. [2-4]). Идея использования матричного метода применительно к задачам с периодическим режимом упоминается в [5], однако из-за громоздких преобразований, которые нужно было бы выполнять вручную и невозможности использования в то время символьных вычислений на ЭВМ матричный метод не получил распространения. В настоящее время, когда существуют различные эффективные системы компьютерной алгебры, матричный метод может быть весьма перспективным как аналитический метод решения задач переноса в многослойных средах. Чтобы охватить единым подходом случаи как плоских слоев, так и искривленных оболочек, совместно с матричным методом используется аппарат обобщенных степеней Берса (см. [1,9]).

Рассмотрим кратко сущность этого подхода применительно к системе

$$
D_{2}^{(i)} D_{1}^{(i)} T^{(i)}(x)=0, \quad i=\overline{1, n} .
$$

Для определенности будем считать, что с возрастанием координаты температура растет. Основной вопрос: произойдет ли фазовый переход в некотором $k$-м слое. Предположим, что на $k$-м слое возможен фазовый переход. Положение возможной границы фаз обозначим $x_{\phi}$. Если фазовый переход на $k$-м слое произошел, то $x_{k}<x_{\phi}<x_{k+1}$; заменим этот слой на два слоя с номерами $k_{1}$ и $k_{2}$ с соответствующими значениями коэффициента теплопроводности $\lambda_{1}{ }^{(k)}$ и $\lambda_{1}{ }^{(k)}$ на них. Таким образом, общее число слоев увеличилось на один.

На сегменте $\left[x_{i}, x_{i+1}\right]$ поставим задачу Коши

$$
\left.T\right|_{x_{i}}=T^{(i)}\left(x_{i}\right),\left.\quad J\right|_{x_{i}}=J^{(i)}\left(x_{i}\right) .
$$

Решение для задачи Коши на сегменте $\left[x_{i}, x_{i+1}\right]$ запишем в виде

$$
T^{(i)}(x)=T^{(i)}\left(x_{i}\right)-X_{i}\left(x, x_{i}\right) J^{(i)}\left(x_{i}\right), \quad J^{(i)}(x)=J^{(i)}\left(x_{i}\right) ;
$$

здесь выражение $X_{i}\left(x, x_{i}\right)$ обозначает первую обобщенную степень Берса с нуль-точкой $x_{i}$. Обобщенная степень определяется соотношением

$$
X_{i}\left(x, x_{i}\right)=\int_{x_{i}}^{x} \frac{d \xi}{a_{1}^{(i)}(\xi)}
$$

следовательно,

$$
D_{1}^{(i)} X_{i}\left(x, x_{i}\right)=a_{1}^{(i)}(x) \frac{d}{d x} \int_{x_{i}}^{x} \frac{d \xi}{a_{1}^{(i)}(\xi)}=1 .
$$

Решение задачи Коши (5) для $i$-го слоя можно записать в матричной форме:

$$
V^{(i)}(x)=K^{(i)}\left(x, x_{i}\right) V^{(i)}\left(x_{i}\right)
$$

где

$$
V^{(i)}(x)=\left(\begin{array}{l}
T^{(i)}(x) \\
J^{(i)}(x)
\end{array}\right), \quad K^{(i)}\left(x, x_{i}\right)=\left(\begin{array}{cc}
1 & -X_{i}^{(1)}\left(x, x_{i}\right) \\
0 & 1
\end{array}\right), \quad V^{(i)}\left(x_{i}\right)=\left(\begin{array}{c}
T^{(i)}\left(x_{i}\right) \\
J^{(i)}\left(x_{i}\right)
\end{array}\right) .
$$

Идеальный контакт слоев (3) в матричной форме принимает вид

$$
V^{(i)}\left(x_{i+1}\right)=V^{(i+1)}\left(x_{i+1}\right) .
$$

Решение для всей системы слоев строится последовательно, начиная с первого слоя. Запишем решение для первого слоя:

$$
V^{(1)}(x)=K^{(1)}\left(x, x_{1}\right) V^{(1)}\left(x_{1}\right), \quad x_{1} \leqslant x \leqslant x_{2} ;
$$


тогда для второго слоя с учетом условий идеального контакта (6) решение примет вид

$$
V^{(2)}(x)=K^{(2)}\left(x, x_{2}\right) V^{(2)}\left(x_{2}\right)=K^{(2)}\left(x, x_{2}\right)\left[K^{(1)}\left(x_{2}, x_{1}\right) V^{(1)}\left(x_{1}\right)\right], \quad x_{2} \leqslant x \leqslant x_{3} .
$$

Выполняя далее по слоям аналогичную последовательную подстановку, получим общий результат для $i$-го слоя:

$$
V^{(i)}(x)=K^{(i)}\left(x, x_{i}\right) K^{(i-1)}\left(x_{i}, x_{i-1}\right) \ldots K^{(1)}\left(x_{2}, x_{1}\right) V^{(1)}\left(x_{1}\right) .
$$

Произведение матриц $K$ для слоев с $j$-го по $i$-й имеет вид

$$
K^{(i, \ldots, j)}\left(x, x_{j}\right)=\left(\begin{array}{c}
1-\left(X_{i}\left(x, x_{i}\right)+\sum_{l=j}^{i-1} X_{l}\left(x_{l+1}, x_{l}\right)\right. \\
1
\end{array}\right), \quad 1 \leqslant j<i .
$$

Физический смысл суммы обобщенных степеней в матрице (8) - это сумма термических сопротивлений слоев при их последовательном соединении.

В конечной точке $x_{n+1}$ системы слоев получим

$$
V^{(n)}\left(x_{n+1}\right)=K^{(n, 1)}\left(x_{n+1}, x_{1}\right) V^{(1)}\left(x_{1}\right) .
$$

Таким образом, формула (9) дает возможность получить решение краевых задач всех трех типов, в общем случае сводя решение к системе двух линейных уравнений при любом конечном числе слоев. Для системы уравнений (2) с краевыми условиями (4) система имеет вид

$$
\left\{\begin{array}{l}
T_{2}=T_{1}-J^{(1)}\left(x_{1}\right) \sum_{i=1}^{n} X_{i}\left(x_{i+1}, x_{i}\right) \\
J^{(n)}\left(x_{n+1}\right)=J^{(1)}\left(x_{1}\right)
\end{array}\right.
$$

Из системы (9) определим значение теплового потока, который в рассматриваемой задаче постоянен для всей многослойной среды; обозначим его $J$ :

$$
J=\frac{T_{1}-T_{2}}{\sum_{i=1}^{n} X_{i}\left(x_{i+1}, x_{i}\right)}
$$

Подставляя значение потока $J$ из (10) в (9), получим решение поставленной задачи $(2),(3),(4)$.

Вернемся к вопросу фазового перехода на $k$-м слое. Запишем для этого слоя систему уравнений

$$
\left\{\begin{array}{l}
T_{\phi}=T_{1}-J\left(X_{k_{1}}\left(x_{\phi}, x_{k}\right)+\sum_{i=1}^{k-1} X_{i}\left(x_{i+1}, x_{i}\right)\right), \\
T_{2}=T_{\phi}-J\left(X_{k_{2}}\left(x_{k+1}, x_{\phi}\right)+\sum_{i=k+1}^{n} X_{i}\left(x_{i+1}, x_{i}\right)\right),
\end{array}\right.
$$

из которой найдем условие для определения координаты границы фаз $x_{\phi}$ :

$$
\frac{\sum_{i=1}^{k-1} X_{i}\left(x_{i+1}, x_{i}\right)+X_{k_{1}}\left(x_{\phi}, x_{k}\right)}{T_{1}-T_{\phi}}=\frac{\sum_{i=k+1}^{n} X_{i}\left(x_{i+1}, x_{i}\right)+X_{k_{2}}\left(x_{k+1}, x_{\phi}\right)}{T_{\phi}-T_{2}}, \quad x_{k}<x_{\phi}<x_{k+1} .
$$

В наиболее простом случае, когда имеем систему из одного материала, очевидно, что фазовый переход возможен только при выполнении условия $T_{1}<T_{\phi}<T_{2}$ (напомним, что для определенности мы считаем, что температура растет с увеличением координаты). В этом случае получаем два слоя с коэффициентами теплопроводности фаз $\lambda_{1}$ и $\lambda_{2}$ соответственно. Запишем согласно (11) уравнение для нахождения координаты точки фазового перехода:

$$
\frac{X_{1}\left(x_{\phi}, x_{1}\right)}{T_{1}-T_{\phi}}=\frac{X_{2}\left(x_{2}, x_{\phi}\right)}{T_{\phi}-T_{2}}, \quad x_{1}<x_{\phi}<x_{2},
$$




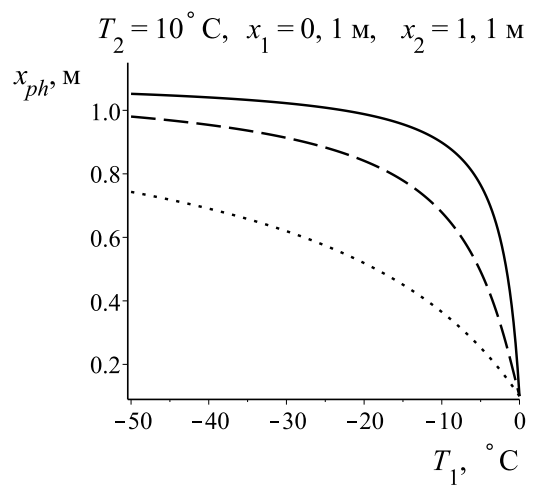

(a)

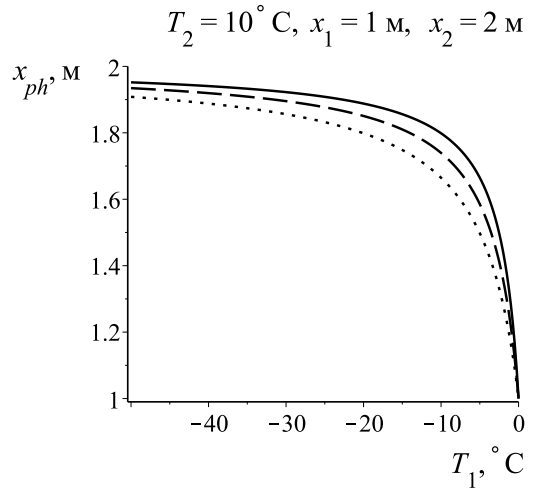

(б)

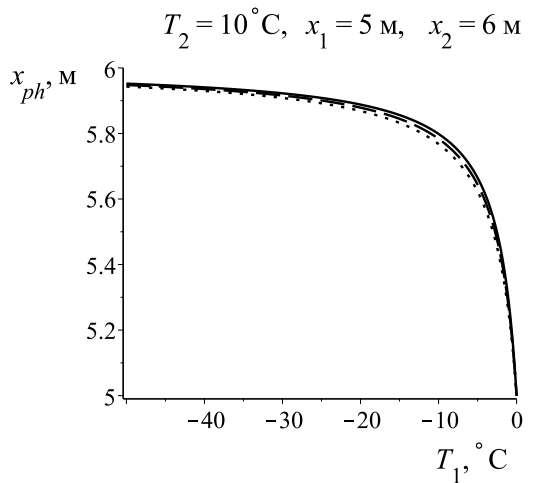

(в)

Рис. 1. Зависимость координаты точки фазового перехода $x_{\phi}$ от внешней температуры $T_{1}$ в системе «лед-вода> для слоев со сдвиговой симметрией (сплошная линия), осевой симметрией (пунктирная линия) и центральной симметрией (точечная линия).

которое для плоских слоев принимает вид

$$
\frac{x_{\phi}-x_{1}}{\lambda_{1}\left(T_{1}-T_{\phi}\right)}=\frac{x_{2}-x_{\phi}}{\lambda_{2}\left(T_{\Phi}-T_{2}\right)},
$$

для слоев с осевой симметрией

$$
\frac{\ln \left(x_{\phi} / x_{1}\right)}{\lambda_{1}\left(T_{1}-T_{\phi}\right)}=\frac{\ln \left(x_{2} / x_{\phi}\right)}{\lambda_{2}\left(T_{\phi}-T_{2}\right)},
$$

и для слоев с центральной симметрией

$$
\frac{x_{\phi}-x_{1}}{\lambda_{1} x_{1}\left(T_{1}-T_{\phi}\right)}=\frac{x_{2}-x_{\phi}}{\lambda_{2} x_{2}\left(T_{\phi}-T_{2}\right)} .
$$

4. Результаты расчетов и их обсуждение. Во всех расчетах для удобства сравнения результатов принято, что температура растет с увеличением координаты, как и было оговорено ранее. Также все расчеты были проведены для случаев сдвиговой, осевой и центральной симметрии системы слоев, и результаты моделирования при одинаковых параметрах выведены на графиках в одной системе координат.

В качестве модельной задачи первоначально была рассмотрена система «лед-вода». Для расчетов теплопроводности льда и воды принимались соответственно равными $\lambda_{1}=2,3 \mathrm{~B}$ / $(\mathrm{м} \cdot \mathrm{K})$ и $\lambda_{2}=0,58 \mathrm{~B}$ т $/($ м $\cdot \mathrm{K})$. Во всех расчетах суммарная толщина системы «лед-вода» принималась равной $1 \mathrm{м}$.

На рис. 1 изображены графики зависимости координаты точки фазового перехода $x_{\phi}$ от внешней температуры $T_{1}$ в системе «лед-вода» при одинаковой внешней температуре $T_{2}=10^{\circ} \mathrm{C}$. Рисунок 1 показывает, что при близком к нулю значению внешней границы $x_{1}$ графики для различных видов симметрии среды сильно различаются: по сравнению с плоской системой для цилиндрических оболочек координата точки фазового перехода находится ближе к «холодной» границе $x_{1}$, а для сферических - еще ближе, что объясняется заметной разницей между площадями внутренней и внешней граничных поверхностей искривленных оболочек. При удалении внутренней координаты от нуля и неизменной толщине среды наблюдается неизменность графика для плоских слоев и приближение к нему графиков для цилиндрических и сферических слоев, что по смыслу соответствует все меньшему различию между площадями внутренних и внешних поверхностей.

На рис. 2 показано влияние увеличения внешней температуры $T_{2}$ на зависимость координаты точки фазового перехода $x_{\phi}$ от внешней температуры $T_{1}$ в системе «лед-вода», при этом координаты внешних границ среды во всех случаях $\left(T_{2}=10^{\circ} \mathrm{C}, T_{2}=30^{\circ} \mathrm{C}, T_{2}=80^{\circ} \mathrm{C}\right)$ рассматривались 


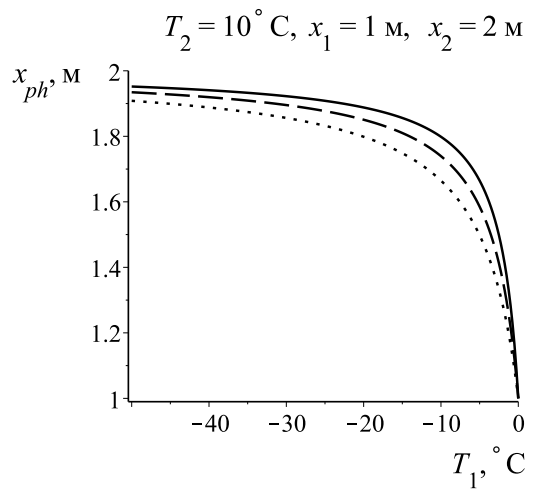

(a)

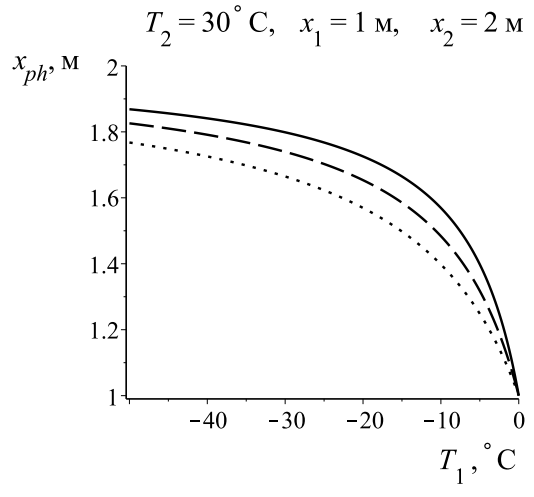

(б)

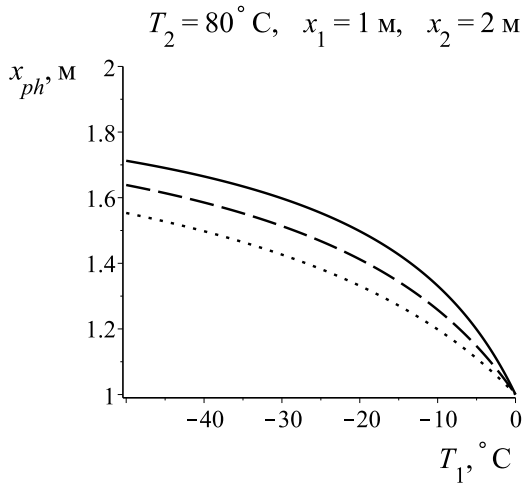

(в)

Рис. 2. Влияние внешней температуры $T_{2}$ на зависимость координаты точки фазового перехода $x_{\phi}$ в системе «лед-вода» для слоев со сдвиговой симметрией (сплошная линия), осевой симметрией (пунктирная линия) и центральной симметрией (точечная линия).

одинаковыми $\left(x_{1}=1 \mathrm{~m}, x_{2}=2 \mathrm{M}\right)$. С увеличением $T_{2}$ точка фазового перехода не только сдвигается ближе к «холодной» границе $x_{1}$, но и заметно меняется характер зависимости координаты $x_{\phi}$ от $T_{1}$ : хорошо выраженный изгиб графиков (рис. $\left.2(\mathrm{a})\right)$ сглаживается, графики становятся более пологими (рис. $2($ б) и $2($ в)).

На рис. 3 показаны распределения температуры, полученные при решении матричным методом первой краевой задачи для системы «лед-вода» при внешних температурах $T_{1}=-20^{\circ} \mathrm{C}$ и $T_{2}=20^{\circ} \mathrm{C}$. На рис. $3\left(\right.$ a) внешние границы координат слоев $x_{1}=0,1 \mathrm{~m}, x_{2}=1,1 \mathrm{м}$. Полученные значения координаты фазового перехода составляют для плоской системы $x_{\phi}=0,899 \mathrm{M}$, для случая осевой симметрии среды $-x_{\phi}=0,679$ м и для центральной симметрии $-x_{\phi}=0,365$ м. Для системы плоских слоев распределение температуры на каждом слое линейное, а для искривленных оболочек хорошо заметна нелинейность распределений на каждом слое. Заметная разница в площадях внутренней и внешней поверхности для искривленной среды приводит к существенному сдвигу координаты фазового перехода к «холодной» границе $x_{1}$. На рис. 3(б) внешние границы координат слоев $x_{1}=1 \mathrm{~m}, x_{2}=2 \mathrm{~m}$, а значения координаты фазового перехода для плоской системы $x_{\phi}=1,799 \mathrm{~m}$, для случая осевой симметрии среды $-x_{\phi}=1,739$ м и для центральной симметрии $-x_{\phi}=1,665$ м. Таким образом, при удалении границы $x_{1}$ от нуля распределение температуры в плоской системе не меняется, а для цилиндрических и сферических слоев в силу уменьшения разницы между площадями внутренней и внешней поверхности графики распределений температуры «выпрямляются», а координата фазового перехода приближается к значению для плоских слоев.

Далее была рассмотрена более сложная модельная задача: система из трех материалов «медьолово-титан» (слои меняются соответственно увеличению координаты границ слоев). На рис. 4 показано распределение температуры в такой системе, полученное при решении первой краевой задачи матричным методом. Заданные внешние температуры $-T_{1}=100^{\circ} \mathrm{C}, T_{2}=650^{\circ} \mathrm{C}$, толщина каждого материала в системе принималась равной 1 м, координаты внешних границ $x_{1}=1 \mathrm{~m}$ и $x_{4}=4 \mathrm{~m}$. Температуры плавления меди $1084,5^{\circ} \mathrm{C}$, олова $231,97^{\circ} \mathrm{C}$, титана $1660^{\circ} \mathrm{C}$. Очевидно, что при заданных внешних температурах фазовый переход возможен только на слое с оловом. При расчетах брались следующие значения теплопроводности материалов: $\lambda_{\mathrm{Cu}}=392 \mathrm{BT} / \mathrm{m} \cdot \mathrm{K}, \lambda_{\mathrm{Sn}}=59,3 \mathrm{~B} \mathrm{~T} /(\mathrm{m} \cdot \mathrm{K})$ для твердой фазы и $\lambda_{\mathrm{Sn}}=31 \mathrm{BT} /(\mathrm{м} \cdot \mathrm{K})$ для жидкой фазы, $\lambda_{\mathrm{Ti}}=19,8 \mathrm{BT} /(\mathrm{M} \cdot \mathrm{K})$. Матричным методом были определены значения координат точек фазового перехода: в системе плоских слоев $x_{\phi}=2,590 \mathrm{~m}$, в системе с осевой симметрией $x_{\phi}=2,340$ м и в системе с центральной симметрией $x_{\phi}=2,110$ м. 


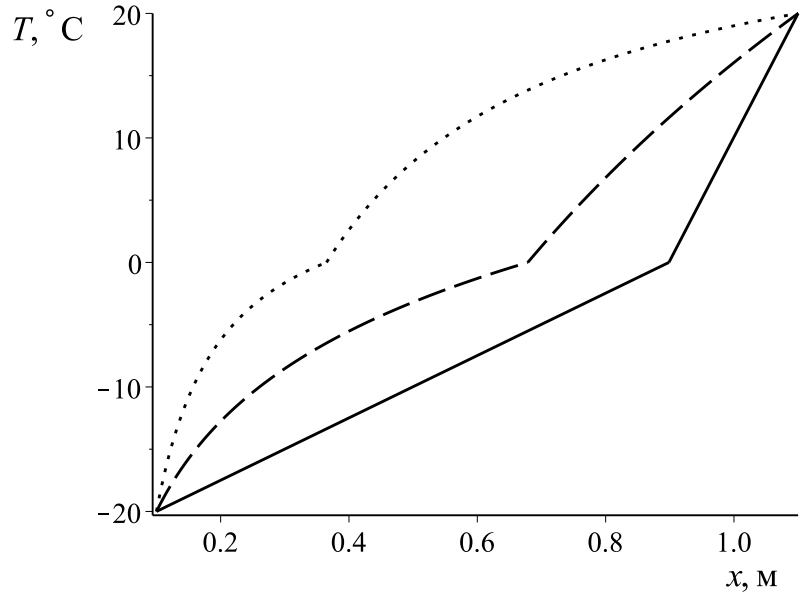

(a)

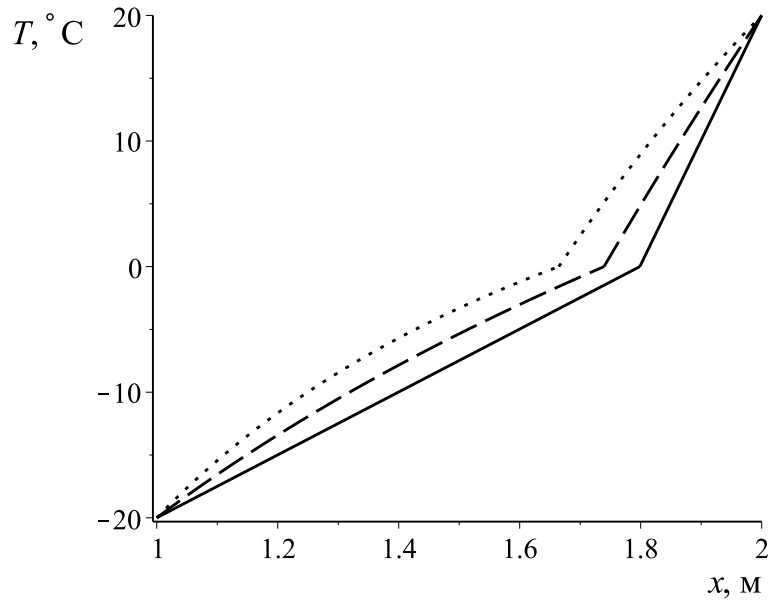

(б)

Рис. 3. Распределение температуры в системе «лед-вода»в плоском (сплошная линия), цилиндрическом (пунктирная линия) и сферическом слое (точечная линия).

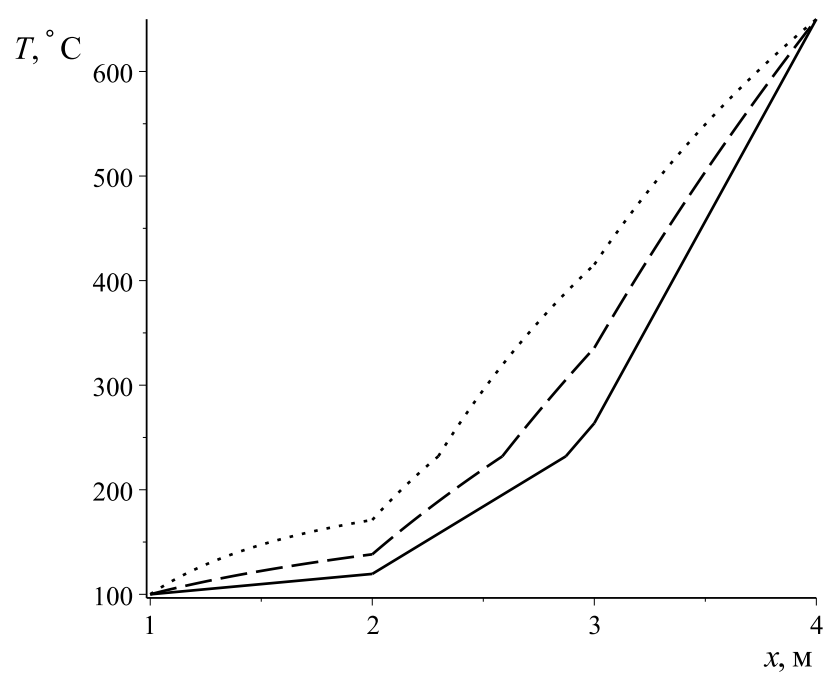

Рис. 4. Распределение температуры в трехслойной системе «медь-олово-титан» при наличии фазового перехода в слое с оловом в среде со сдвиговой симметрией (сплошная линия), с осевой симметрией (пунктирная линия) и с центральной симметрией (точечная линия).

5. Заключение. Рассмотренный в работе матричный метод позволяет сравнительно несложно аналитически получить решение задачи теплопроводности в многослойной среде при наличии фазовых переходов. В работе подробно рассмотрено решение первой краевой задачи однородного уравнения теплопроводности в среде с возможным фазовым переходом, приведены результаты расчетов по нахождению координаты точки фазового перехода и построению распределения температуры в среде с учетом наличия фазового перехода. Расчеты проведены для многослойной среды со сдвиговой, осевой и центральной симметрией. Показано, что возможно решение предлагаемым методом и краевых задач второго и третьего типа. Матричный метод позволяет также решать задачи и с более сложными условиями, например, наличие внутренних источников тепла или неоднородность слоев в зависимости от координаты. Таким образом, этот метод может 
быть довольно эффективным для решения различных практических задач, например, когда важно определить условия нежелательного фазового перехода при проектировании и эксплуатации различных технических устройств.

\section{СПИСОК ЛИТЕРАТУРЫ}

1. Гладышев Ю. А. Метод обобщенных степеней Берса и его приложение в математической физике. Калуга: КГУ им. К. Э. Циолковского, 2011.

2. Гладышев Ю. А., Калманович В. В., Серегина Е. В., Степович М. А. О возможности совместного применения матричного метода и аппарата обобщенных степеней Берса для математического моделирования процесса теплопереноса в объектах, обладающих цилиндрической симметрией// Вопросы атомной науки и техники. Сер. Ядерно-реакторные константы. - 2018. - № 3. - С. 158-167.

3. Гладышев Ю. А., Калманович В. В., Степович М. А. О возможности приложения аппарата Берса к моделированию процессов тепломассопереноса, обусловленного электронами в планарной многослойной среде// Поверхность. Рентген. синхротрон. нейтрон. исслед. - 2017. - № 10. - С. 105-110.

4. Калманович B. B., Степович M. A. О совместном применении матричного метода и аппарата обобщенных степеней Берса для математического моделирования процессов тепломассопереноса в полупроводниковых материалах электронной техники// в кн.: Проблемы разработки перспективных микро- и наноэлектронных систем. - М.: ИППМ РАН, 2018. - С. 194-201.

5. Карслоу Г., Егер Д. Теплопроводность твердых тел. - М.: Наука, 1964.

6. Карташов Э. М. Аналитические методы в теории теплопроводности твердых тел. - М.: Высшая школа, 2001.

7. Кудинов В. А., Карташов Э. М., Калашников В. В. Аналитические решения задач тепломассопереноса и термоупругости для многослойных конструкций. - М.: Высшая школа, 2005.

8. Лъков А. В. Теория теплопроводности. - М.: Высшая школа, 1967.

9. Bers L., Gelbart A. On a class of functions defined by partial differential equations// Trans. Am. Math. Soc. - 1944. - 56. - P. 67-93.

Гладышев Юрий Александрович

Калужский государственный университет им. К. Э. Циолковского

E-mail: v572264@yandex.ru

Калманович Вероника Валерьевна

Калужский государственный университет им. К. Э. Циолковского

E-mail: v572264@yandex.ru 